\title{
Diagnosing features of Jacobson's nerve schwannoma
}

\author{
Amit $\underline{K a r a n d i k a r}^{1}$, FRCR, Tiong Yong $\underline{\operatorname{Tan}}^{2}$, FRCR, Raymond YS $\underline{\mathrm{NgO}}^{3}$, FAMS
}

\begin{abstract}
Jacobson's nerve schwannoma is a rare middle ear tumour presenting as a mass along the cochlear promontory. It can be differentiated from other masses along the promontory based on clinical findings, and computed tomography and magnetic resonance imaging features. For small-sized masses, it is possible to differentiate the various middle ear schwannomas from one another. We present the case of a 40-year-old woman with a ten-year history of left hearing loss who was diagnosed with Jacobson's nerve schwannoma.
\end{abstract}

Keywords: glomus tympanicum, Jacobson's, schwannoma

\section{INTRODUCTION}

Jacobson's nerve schwannoma is a rare middle ear tumour. Jacobson's nerve is a tympanic branch of the glossopharyngeal nerve, arising from its inferior ganglion. It enters the middle ear cavity through the inferior tympanic canaliculus, runs in a canal on the cochlear promontory and provides the main sensory innervation to the mucosa of the mesotympanum and Eustachian tube. It then joins the caroticotympanic nerve to form the lesser superficial petrosal nerve.

In this study, we report the case of a 40-year-old woman with a ten-year history of left hearing loss who was diagnosed with Jacobson's nerve schwannoma based on clinical findings and imaging features.

\section{CASE REPORT}

A 40-year-old Chinese woman presented with left hearing loss for ten years. There was no otalgia, tinnitus, giddiness, otorrhoea or facial palsy. The patient's physical examination revealed a pale, non-pulsatile, soft tissue mass in the middle ear, with a bulging tympanic membrane. A pure tone audiogram showed severe left-sided sensorineural hearing loss. Computed tomography (CT) of the temporal bone showed a lobulated soft tissue mass in the left middle ear (Fig. 1). The mass was based on the cochlear promontory and measured $1.0 \mathrm{~cm} \times 0.8 \mathrm{~cm} \times 0.8 \mathrm{~cm}$ in size. The CT image also showed displacement of the malleus and incus laterally, with destruction of the long process of the incus, stapes superstructure and footplate of the stapes. The mass had eroded the promontory, oval window and round window niche. The facial nerve canal was intact and distinct from the mass (Fig. 2). The left inferior tympanic canaliculus was widened when compared to the right side (Fig. 3). Magnetic resonance (MR) imaging showed a significantly enhancing mass in the middle ear extending into the basal turn of the cochlea and vestibule (Fig. 4).

Given the clinical findings, location, enlarged inferior tympanic canaliculus and enhancement of the mass, a diagnosis of Jacobson's nerve schwannoma was made. An exploratory tympanotomy with biopsy of the middle ear mass confirmed the

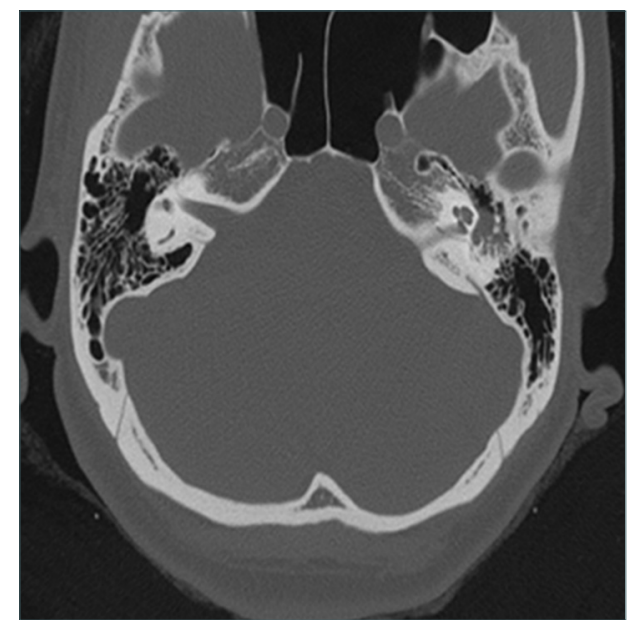

Fig. 1 CT image of the temporal bone shows a soft tissue mass displacing the head of the malleus laterally and eroding into the vestibule in the left middle ear.

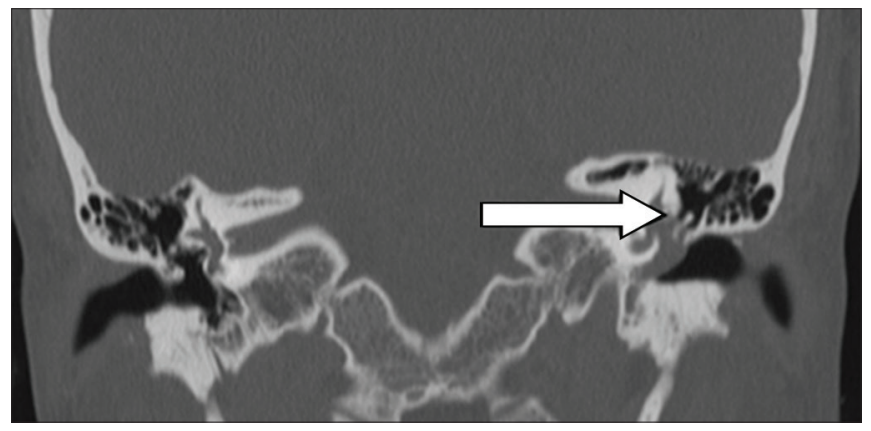

Fig. 2 CT image of the temporal bone shows a mass based on the cochlear promontory on the left side. The facial nerve canal is seen separate from the mass (arrow).

diagnosis. The patient refused surgery and was therefore treated conservatively. Follow-up MR imaging after four years showed interval stability with no new symptoms.

\section{DISCUSSION}

Our patient presented with severe left-sided severe sensorineural hearing loss and a pale, non-pulsatile, soft tissue mass in the middle ear on physical examination. In our patient, a diagnosis of Jacobson's nerve schwannoma was made based on the location of

${ }^{1}$ Department of Diagnostic Radiology, Tan Tock Seng Hospital, ${ }^{2}$ Department of Radiology, Changi General Hospital, ${ }^{3}$ Department of Otolaryngology-Head and Neck Surgery, National University Hospital, Singapore

Correspondence: Dr Amit Karandikar, Consultant, Department of Radiology, Tan Tock Seng Hospital, 11 Jalan, Tan Tock Seng, Singapore 308433.

amit_karandikar@ttsh.com.sg 

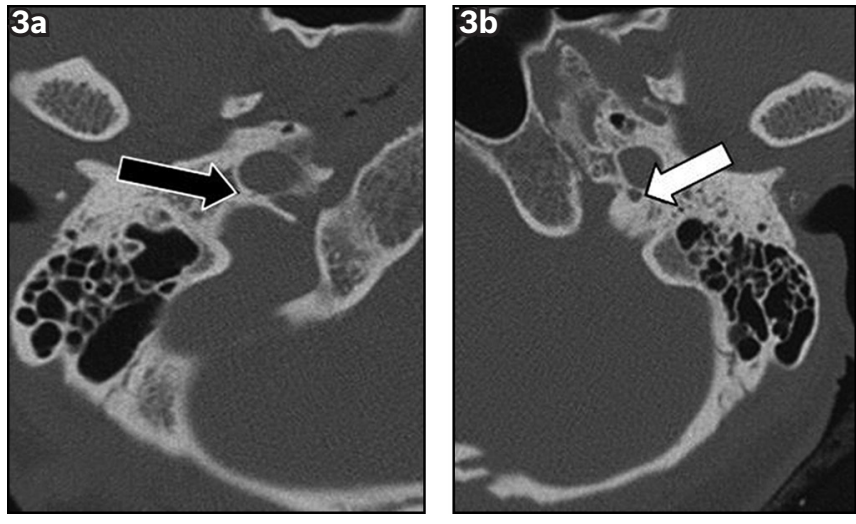

Fig. 3 CT images of the temporal bone show (a) a normal right inferior tympanic canaliculus (black arrow) and (b) an enlarged left inferior tympanic canaliculus (white arrow). Although the images were taken at different levels, the difference in sizes is apparent.

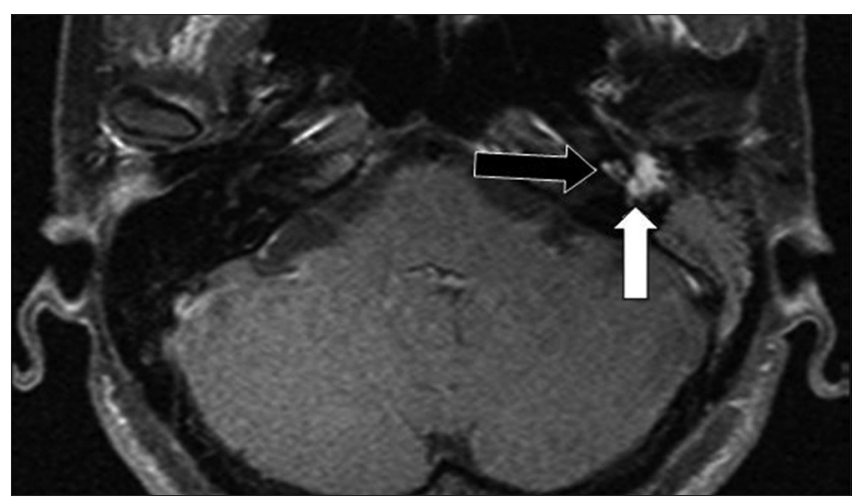

Fig. 4 Axial T1-W contrast-enhanced MR image shows an enhancing middle ea mass on the left side. Enhancement was also seen in the left inner ear structures such as the vestibule (white arrow) and cochlea (black arrow).

the mass, clinical findings and imaging features. The sensorineural hearing deficit seen in our patient was thought to be caused by invasion into the inner ear structures.

Several lesions need to be included in the differential diagnosis of Jacobson's nerve schwannoma. The most common tumour seen at the cochlear promontory is the glomus tympanicum. ${ }^{(1}$ Other less common causes include congenital cholesteatoma and Jacobson's nerve schwannoma. ${ }^{(2)}$

Although glomus tympanicum and Jacobson's nerve schwannoma are similar in location, distinct imaging and clinical features help to differentiate the two lesions. On CT/MR imaging, glomus tympanicum is seen as an intensely enhancing mass based on the cochlear promontory. ${ }^{(3)}$ On the other hand, radiologically, Jacobson's nerve schwannoma appears on CT as an expansile soft tissue mass without significant enhancement. ${ }^{(4)}$ The inferior tympanic canaliculus can be identified on CT anterior to the internal jugular vein. (1) On MR imaging, Jacobson's nerve schwannoma is hypointense on T1-weighted and hyperintense on T2-weighted images, showing homogeneous enhancement. ${ }^{(4)}$ Erosion of the cochlear promontory may be seen. ${ }^{(1)}$ In other words, glomus tympanicum is seen otoscopically as a red, pulsatile mass with intense enhancement on CT/MR imaging while Jacobson's nerve schwannoma presents as a pale, non-pulsatile mass showing moderate enhancement on MR imaging but appearing without enhancement on CT.

Of all middle ear schwannomas, the facial nerve schwannoma is the most common entity. ${ }^{(2)}$ They present expansile masses along the facial nerve canal, with the most common location being the geniculate ganglion. ${ }^{(5)}$ Other middle ear schwannomas are rare and may arise from the chorda tympani and Jacobson's nerve. ${ }^{(2)}$

The signal characteristics and enhancement features of Jacobson's nerve schwannoma are similar to those of facial nerve schwannoma. However, the location of the mass on the cochlear promontory, presence of an enlarged inferior tympanic canaliculus and a distinctly separate facial nerve canal help to differentiate Jacobson's nerve schwannoma from facial nerve schwannoma. ${ }^{(1)}$ In similar fashion, Jacobson's nerve schwannomas can be differentiated from chorda tympani nerve schwannomas, which are located along the course of the corresponding nerve. ${ }^{(6)}$

During the differential diagnosis of the lesion, it is also important to distinguish between Jacobson's nerve schwannoma and non-tumour conditions at the cochlear promontory, such as congenital cholesteatoma that may exhibit restricted diffusion and lack of enhancement on MR imaging. ${ }^{(7)}$

In conclusion, Jacobson's nerve schwannoma typically presents as a mass along the cochlear promontory with an enlarged inferior tympanic canaliculus. Imaging findings on CT and MR imaging can help to differentiate it from the various other masses that may be seen on the cochlear promontory such as glomus tympanicum and congenital cholesteatoma. While facial nerve schwannoma is the most common schwannoma, it is possible to differentiate the various middle ear schwanommas from one another based on the location and course of the mass. Here, we presented the case of a 40-year-old woman with a ten-year history of left hearing loss who was diagnosed with Jacobson's nerve schwannoma based on tumour location, clinical findings and imaging features. Interval stability was seen in our patient who, having refused surgery, was managed conservatively at the four-year follow-up.

\section{REFERENCES}

1. Aydin K, Maya MM, Lo WW, Brackmann DE, Kesser B. Jacobson's nerve schwannoma presenting as a middle ear mass. AJNR Am J Neuroradiol 2000; 21:1331-3.

2. Kim CW, Han DH, Kim CH, Cho SJ, Rho YS. Primary middle ear schwannoma. Am J Otolaryngol 2007, 28:342-6.

3. Lee KY, Oh YW, Noh HJ, et al. Extraadrenal paragangliomas of the body: imaging features. AJR Am J Roentgenol 2006; 187:492-504.

4. Wiggins RH 3rd, Harnsberger HR, Salzman KL, et al. The many faces of facial nerve schwannoma. AJNR Am J Neuroradiol 2006; 27:694-9.

5. Kertesz TR, Shelton C, Wiggins $\mathrm{RH}$, et al. Intratemporal facial nerve neuroma: anatomical and radiological features. Laryngoscope 2001; 111:1250-6.

6. Roig O R JL, Roig-Ocampos F JL, Serafini DP, Lopez Filho O. [Middle ear schwannoma]. Braz J Otorhinolaryngol 2010; 76:673. Portuguese.

7. Baráth K, Huber AM, Stämpfli P, Varga Z, Kollias S. Neuroradiology of cholesteatomas. AJNR Am J Neuroradiol 2011; 32:221-9. 